\title{
High-pressure phases of uranium monophosphide studied by synchrotron x-ray diffraction
}

\author{
J. Staun Olsen \\ Physics Laboratory, University of Copenhagen, Universitetsparken 5, DK-2100 Copenhagen, Denmark \\ L. Gerward \\ Laboratory of Applied Physics III, Technical University of Denmark, DK-2800 Lyngby, Denmark \\ U. Benedict and S. Dabos \\ Commission of the European Communities, Joint Research Centre, European Institute for Transuranium Elements, Postfach 2340, \\ D-7500 Karlsruhe, Federal Republic of Germany \\ O. Vogt \\ Eidgenössische Technische Hochschule, Laboratorium für Festkörperphysik, Hönggerberg, CH-8093 Zürich, Switzerland
}

(Received 18 August 1987; revised manuscript received 14 December 1987)

\begin{abstract}
X-ray diffraction studies have been performed on UP powder for pressures up to $51 \mathrm{GPa}$ using synchrotron radiation and a diamond-anvil cell. At ambient pressure UP has the rocksalt structure. The bulk modulus has been determined to $B_{0}=102(4) \mathrm{GPa}$ and its pressure derivative to $B_{0}^{\prime}=4.0(8)$. The cubic phase has been found to transform to a new phase, UP II, at about $10 \mathrm{GPa}$. UP II can be characterized by a rhombohedral Bravais lattice. UP II transforms to an orthorhombic phase, UP III, at $28 \mathrm{GPa}$. No volume change has been observed at the two transitions. The influence of the $5 f$ electrons on the transformations is discussed.
\end{abstract}

\section{INTRODUCTION}

The present work is part of a series of experiments to study the high-pressure structural behavior of uranium and thorium compounds containing elements from the IV $A, V A$, and VI $A$ groups of the Periodic Table. UP, like many of these compounds, has the rocksalt structure $(B 1)$ at normal pressure and temperature. At low temperature UP is antiferromagnetic, the Néel temperature $\left(T_{N)}\right.$ being $123 \mathrm{~K}$. No structural change is observed when lowering the temperature. In contrast, US, for example, is ferromagnetic with a Curie temperature $\left(T_{c}\right)$ of $177 \mathrm{~K}$, and a phase transformation to a rhombohederal structure occurs at lower temperatures.

The high-pressure structural behavior of UP has been studied by Vaidya et al. ${ }^{1}$ up to $18 \mathrm{GPa}$ and by Leger et $a l .^{2}$ up to $25 \mathrm{GPa}$. In the present work we report measurements in the extended-pressure range up to $51 \mathrm{GPa}$ where two phase transitions, similar to those for US (Ref. 3) and $U N,{ }^{4}$ are observed.

\section{EXPERIMENTAL PROCEDURE}

Uranium monophosphide, UP, was prepared by direct reaction of stoichiometric amounts of the constituents in a sealed tungsten container. ${ }^{5} \mathrm{X}$-ray diffraction studies were performed at DESY-HASYLAB, Hamburg, West Germany, using synchrotron radiation from the storage ring DORIS II. The electron energy was $3.7 \mathrm{GeV}$ in runs dedicated for radiation work and $5.2 \mathrm{GeV}$ in runs for high-energy physics. The diffraction spectra were recorded using the energy-dispersive technique. Experimental details have been published elsewhere. ${ }^{4}$

High pressure up to $51 \mathrm{GPa}$ has been generated in a diamond-anvil cell where an Inconel gasket enclosed the fine UP powder, the ruby pressure marker, and the pressure-transmitting medium. The latter was either a 4:1 methanol-ethanol mixture or nitrogen. Okai et al. ${ }^{6}$ have analyzed different liquid and solid transmitting media and come to the conclusion that the $4: 1$ methanol:ethanol mixture remains hydrostatic at least up to $20 \mathrm{GPa}$, i.e., even after the solidification at about 11 $\mathrm{GPa}$. It has been observed ${ }^{7}$ that nitrogen is nearly hydrostatic up to about $13 \mathrm{GPa}$. Accordingly, in some of our experiments we have loaded the pressure cell in liquid nitrogen.

The pressure was determined by the ruby-fluorescence method using the nonlinear relation ${ }^{8}$

$$
P=(380.8 \mathrm{GPa})\left[\left(1+\Delta \lambda / \lambda_{0}\right)^{5}-1\right],
$$

where $P$ is the pressure, $\lambda_{0}=6942.4 \AA$ is the wavelength of the ruby $R$ line at ambient pressure, and $\Delta \lambda$ is the pressure-induced wavelength shift.

\section{RESULTS}

The lattice constant $a_{0}$ of cubic UP at ambient pressure and room temperature was determined by highprecision powder diffractometry. The result is

$$
a_{0}=5.5844(5) \AA \text {. }
$$

Figure 1 shows examples of energy-dispersive diffraction spectra with the high-pressure cell. The spacing, $d$, of the lattice planes can be calculated from the energies of the corresponding diffraction peaks knowing the Bragg angle. The latter was determined from a $\mathrm{NaCl}$ spectrum. The observed $d$ spacings as functions of pressure are shown in Fig. 2. Three pressure regions can be distinguished. The diffraction lines of the cubic phase ex- 

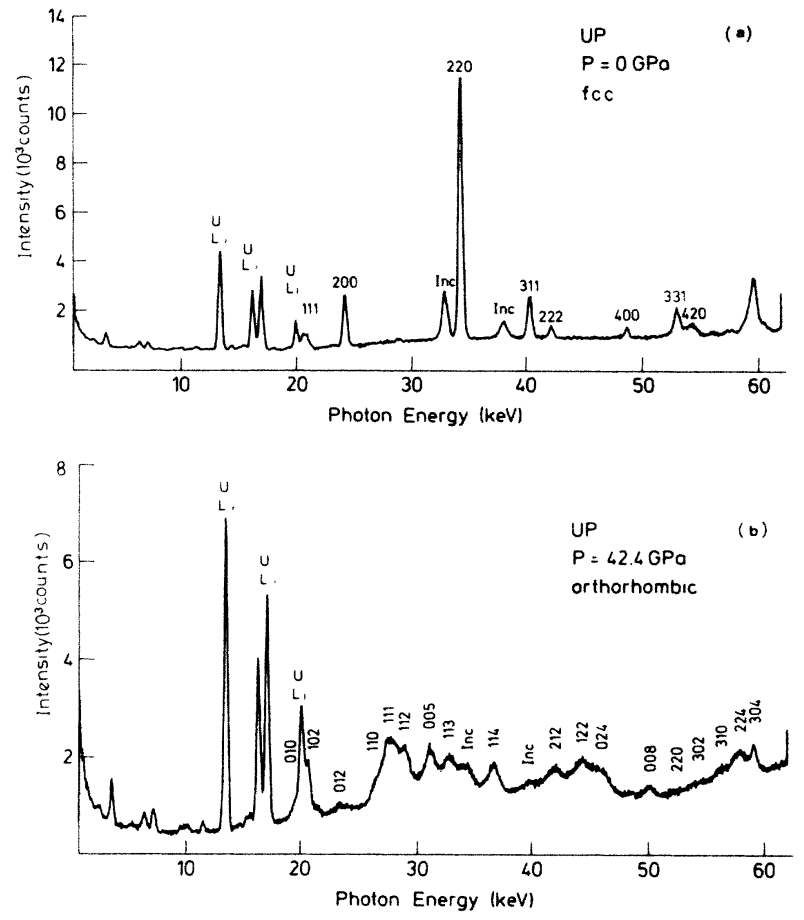

FIG. 1. X-ray energy-dispersive power-diffraction spectra of UP. (a) At atmospheric pressure: fcc phase. (b) At a pressure of $42.4 \mathrm{GPa}$ : orthorhombic phase. Diffraction peaks from the Inconel gasket are denoted Inc. Bragg angle $\theta=5.25^{\circ}$.

tend to about $10 \mathrm{GPa}$. Between 10 and $28 \mathrm{GPa}$ some lines split. Most of the $d$ curves change their slope at 10 $\mathrm{GPa}$, the (200) and (400) curves being notable exceptions. Above $28 \mathrm{GPa}$ several new lines appear. We will denote the two noncubic high-pressure phases UP II and UP III, respectively.

The diffraction lines of UP II can be indexed assuming a rhombohedral distortion of the fcc lattice. Table I lists the calculated and observed $d$ spacings at $16.6 \mathrm{GPa}$. The goodness of the fit is characterized by a reliability factor $R=0.23 \%$ as defined in Table $\mathrm{I}$. The $R$ value is considered highly satisfactory for a high-pressure energydispersive spectrum. Each of the (111) and (220) lines of the cubic phase splits into a pair of lines as seen in Table I and Fig. 2. The separation on the photon-energy scale of the two components of a doublet is less than or equal to the breadth of each line (measured as the full width at half maximum for an $x$-ray fluorescence line in the same energy range). Therefore, the doublets appear as broad and asymmetric peaks in the raw data. The component lines of the doublets have been resolved by a deconvolution technique using the maximum-entropy principle. ${ }^{10}$ Another characteristic feature of the rhombohedral distortion is that it has a negligible effect on the $d$ values of the $(h 00)$ planes, which explains why the (200) and (400) curves in Fig. 2 are unaffected by the phase transition.

The diffraction lines of UP III have been indexed using an orthorhombic unit cell where $c / a \approx c / b \approx 2 \sqrt{2}$, which can be considered as another distorted fcc structure. ${ }^{11}$ The doubling of the $c$ axis has been done in order to ex-
TABLE I. Rhombohedrally distorted UP at $16.6 \mathrm{GPa}$ : $a=5.388(13) \AA, \alpha=89.4(2)^{\circ}$. The indices $h k l$ refer to a facecentered rhombohedral unit cell in order to facilitate the comparison with the fcc phase. The indices $H K L$ refer to the conventional triple-hexagonal unit cell (space group $R \overline{3} m$, no. 166). The calculated $d$ spacings have been obtained using the refinement procedure PURUM (Ref. 9). Intensity labels: s denotes strong, $\mathrm{m}$ denotes medium, $\mathrm{w}$ denotes weak, $\mathrm{vw}$ denotes very weak, and $\mathrm{t}$ denotes trace (shoulder on a broad peak).

\begin{tabular}{ccccc}
\hline \hline$H K L$ & $h k l$ & $d_{\text {calc }}(\AA)$ & $d_{\text {obs }}(\AA)$ & Intensity \\
\hline 003 & 111 & 3.141 & 3.145 & $\mathrm{~m}$ \\
101 & $1 \overline{1} 1$ & 3.101 & 3.110 & $\mathrm{t}$ \\
102 & 200 & 2.694 & 2.687 & $\mathrm{~s}$ \\
104 & 220 & 1.914 & 1.924 & $\mathrm{~m}$ \\
110 & $2 \overline{2} 0$ & 1.896 & 1.900 & $\mathrm{t}$ \\
105 & 311 & 1.635 & 1.631 & $\mathrm{~m}$ \\
113 & $\overline{1} 13$ & 1.623 & & \\
201 & $\overline{3} 11$ & 1.617 & & \\
006 & 222 & 1.571 & 1.569 & $\mathrm{w}$ \\
202 & $2 \overline{2} 2$ & 1.550 & & \\
204 & 400 & 1.347 & 1.343 & $\mathrm{w}$ \\
107 & 331 & 1.245 & 1.243 & $\mathrm{w}$ \\
205 & $\overline{1} 33$ & 1.238 & & \\
211 & $\overline{3} 31$ & 1.230 & & $\mathrm{vw}$ \\
116 & 420 & 1.209 & 1.208 & \\
122 & $\overline{4} 20$ & 1.200 & & \\
\multicolumn{5}{|c|}{} \\
\hline \hline
\end{tabular}

plain some of the new lines that are not merely a splitting of the fcc lines. Table II lists calculated and observed $d$ spacings at $42.4 \mathrm{GPa}$. The $R$ factor of $0.85 \%$ is larger than for UP II because of the poorer diffraction data. Therefore, the proposed orthorhombic structure should be considered as an indexing scheme at present, indicating the position of the structure in the symmetry hierar-

TABLE II. Orthorhombic UP III at $42.4 \mathrm{GPa}: a=3.79(4)$ $\AA, b=3.47(2) \AA, c=10.75(2) \AA$. For intensities, see Fig. 1(b).

\begin{tabular}{lcc}
\hline$h k l$ & $d_{\text {calc }}(\AA)$ & $d_{\text {obs }}(\AA)$ \\
\hline 010 & 3.470 & 3.480 \\
102 & 3.102 & 3.146 \\
012 & 2.916 & 2.912 \\
110 & 2.562 & 2.569 \\
111 & 2.492 & 2.434 \\
112 & 2.313 & 2.343 \\
005 & 2.151 & 2.170 \\
113 & 2.084 & 2.056 \\
114 & 1.855 & 1.851 \\
202 & 1.791 & 1.777 \\
212 & 1.591 & 1.615 \\
122 & 1.514 & 1.515 \\
024 & 1.458 & 1.470 \\
008 & 1.344 & 1.346 \\
310 & 1.189 & 1.207 \\
224 & 1.156 & 1.172 \\
304 & 1.145 & 1.146 \\
& $R=100 \sum\left|d_{\text {calc }}-d_{\text {obs }}\right| / \sum d_{\text {obs }}=0.85 \%$
\end{tabular}


chy. ${ }^{11}$ A similar situation has been found for some other distorted fcc structures suggested for the rare-earths and the actinides. ${ }^{12}$ Table III lists the unit-cell dimensions for all measured pressures.

The pressure dependence of the unit-cell volume can be described by a semiempirical equation of state. The experimental data for the cubic phase have been fitted to the Murnaghan and Birch first-order equations, ${ }^{13}$ respectively. The fitting parameters are $B_{0}$ and $B_{0}^{\prime}$, i.e., the isothermal bulk modulus at ambient pressure and its pressure derivative. The Murnaghan equation gives $B_{0}$ $=102.5 \mathrm{GPa}$ and $B_{0}^{\prime}=3.9$, the Birch equation $B_{0}=102.1 \mathrm{GPa}$ and $B_{0}^{\prime}=4$. 1. As a final result we quote

$$
B_{0}=102(4) \mathrm{GPa}, \quad B_{0}^{\prime}=4.0(8) \text {, }
$$

where the uncertainties are the standard deviations of the least-squares fit to the equation of state.

\section{DISCUSSION}

\section{A. Diffraction data}

The lattice constant $a_{0}$ of cubic UP depends on the stoichiometry. A comparison with published data (Table
IV) indicates that our specimen has the composition $\mathbf{U P}_{x}$, where $x=0.98-0.99$. The theoretical $a_{0}$ value, ${ }^{17} \mathrm{calcu}-$ lated at $0 \mathrm{~K}$, is $2 \%$ lower than the experimental value. Similar differences are found for other actinide rocksaltstructure compounds.

Table $\mathbf{V}$ compares published data for the bulk modulus $\boldsymbol{B}_{0}$ and its pressure derivative $\boldsymbol{B}_{0}^{\prime}$. It is seen that our result is in good agreement with the experimental result of Leger et al., ${ }^{2}$ and with the theoretical calculation of Brooks. ${ }^{17}$ The experimental $B_{0}$ value reported by Vaidya et al. ${ }^{1}$ seems to be anomalously high. The pressurevolume data given by these authors correspond to a negative $B_{0}^{\prime}$ value which is unlikely.

The rhombohedral distortion has been observed previously by Leger et al. ${ }^{2}$ These authors suggest that one is observing an effect of uniaxial-stress components due to the freezing of the 4:1 methanol-ethanol liquid in the pressure range 10-15 GPa. According to the model devised by Singh and Balasingh, ${ }^{18}$ the $d$ spacings are then modified similarly to a rhombohedral distortion. Compared with other uranium compounds UP would be unusually sensitive to uniaxial stress.

It follows from above that one cannot distinguish between a real rhombohedral phase and an apparent distor-

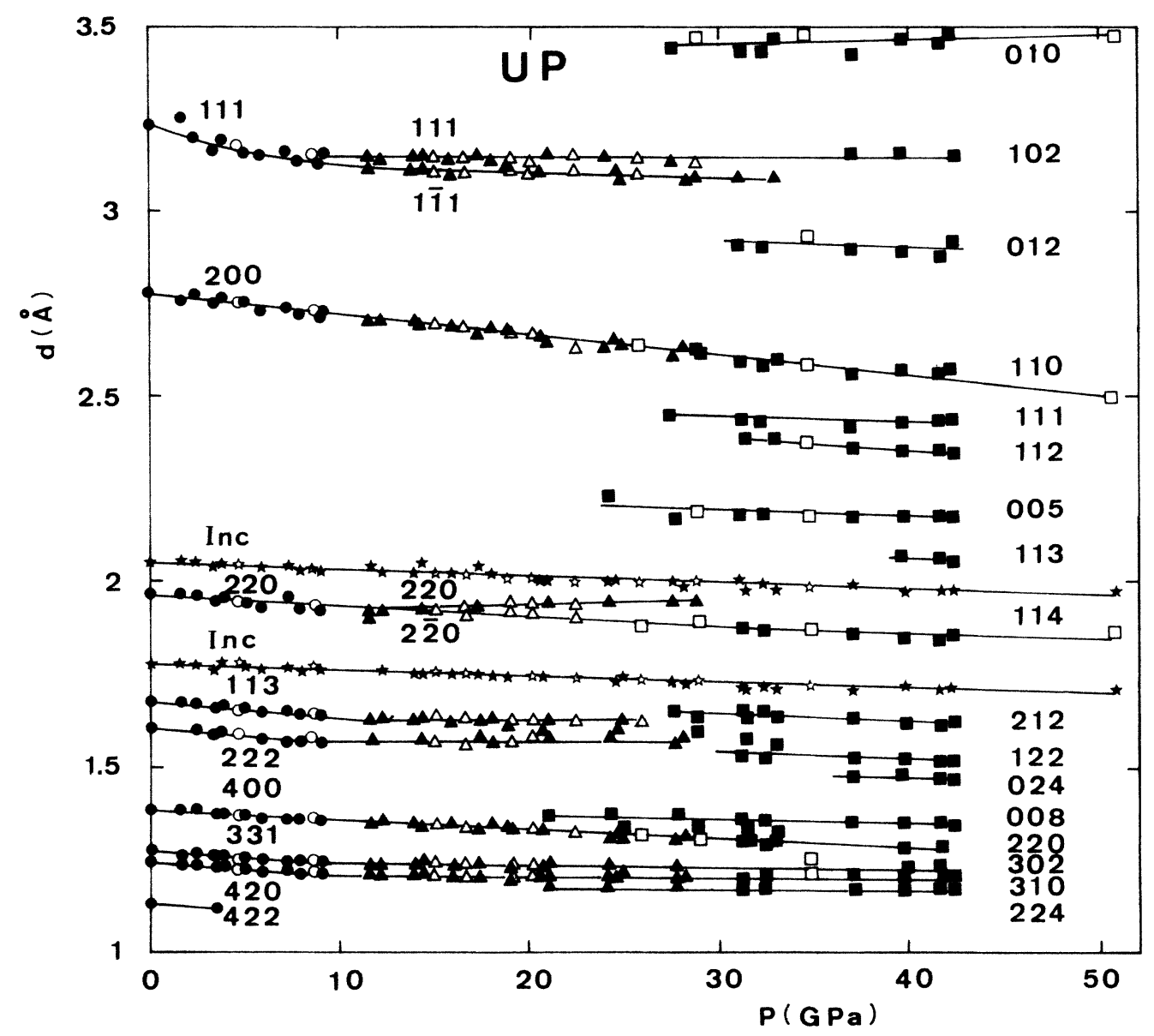

FIG. 2. Interplanar spacings as functions of pressure. The indices based on the fcc unit cell are shown on the left-hand side; those based on the orthorhombic unit cell are on the right-hand side. Circles denote the cubic phase, triangles the rhombohedral phase, squares the orthorhombic phase, and stars the Inconel lines of the gasket. Open symbols refer to the use of nitrogen as the pressuretransmitting medium. 
tion due to uniaxial-stress components in the methanolethanol mixture. Therefore, we have also made a series of experiments with nitrogen as the pressure-transmitting medium. This should ensure truly hydrostatic pressure in the pressure range of interest here. As seen in Fig. 2, the experimental $d$ values are reproducible and we conclude that we are observing a real phase transformation to rhombohedral UP II.

TABLE III. Unit-cell dimensions for UP. The uncertainties given in parentheses are the standard deviations of the leastsquares fit.

\begin{tabular}{|c|c|}
\hline$P(\mathrm{GPa})$ & $a(\AA)$ \\
\hline \multicolumn{2}{|c|}{ Face-centered cubic } \\
\hline 0 & $5.5844(5)^{\mathrm{a}}$ \\
\hline 0 & $5.581(5)$ \\
\hline 2.45 & $5.542(5)$ \\
\hline 3.77 & $5.518(3)$ \\
\hline 4.55 & $5.508(9)$ \\
\hline 5.07 & $5.496(3)$ \\
\hline 5.94 & $5.481(4)$ \\
\hline 7.24 & $5.465(6)$ \\
\hline 7.86 & $5.455(8)$ \\
\hline 8.98 & $5.446(12)$ \\
\hline 9.20 & $5.440(12)$ \\
\hline
\end{tabular}

$a(\AA)$ $\alpha(\mathrm{deg})$

\begin{tabular}{|c|c|c|c|}
\hline \multicolumn{3}{|c|}{$a(\mathrm{~A})$} & $\alpha$ (deg) \\
\hline \multicolumn{4}{|c|}{ Rhombohedral } \\
\hline 11.6 & \multicolumn{2}{|c|}{$5.41(1)$} & $89.4(1)$ \\
\hline 13.9 & \multicolumn{2}{|c|}{$5.40(1)$} & $89.5(2)$ \\
\hline 14.3 & \multicolumn{2}{|c|}{$5.39(1)$} & $89.2(2)$ \\
\hline 15.0 & \multicolumn{2}{|c|}{$5.41(2)$} & $89.6(2)$ \\
\hline 15.9 & \multicolumn{2}{|c|}{$5.38(2)$} & $89.4(5)$ \\
\hline 16.6 & \multicolumn{2}{|c|}{$5.39(2)$} & $89.4(2)$ \\
\hline 17.0 & \multicolumn{2}{|c|}{$5.38(2)$} & $89.2(2)$ \\
\hline 17.3 & \multicolumn{2}{|c|}{$5.36(2)$} & $88.7(3)$ \\
\hline 18.8 & \multicolumn{2}{|c|}{$5.35(2)$} & $89.3(2)$ \\
\hline 19.0 & \multicolumn{2}{|c|}{$5.36(2)$} & $89.2(2)$ \\
\hline 20.1 & \multicolumn{2}{|c|}{$5.36(2)$} & $88.6(3)$ \\
\hline 21.1 & \multicolumn{2}{|c|}{$5.34(3)$} & $88.3(5)$ \\
\hline 22.2 & \multicolumn{2}{|c|}{$5.34(5)$} & $89.0(9)$ \\
\hline \multirow[t]{2}{*}{24.1} & \multicolumn{2}{|c|}{$5.32(4)$} & $88.1(7)$ \\
\hline & $a(\AA)$ & $b(\AA ̊ \AA)$ & $c(\AA)$ \\
\hline \multicolumn{4}{|c|}{ Orthorhombic } \\
\hline 27.6 & $3.85(2)$ & $3.55(3)$ & $10.95(4)$ \\
\hline 28.9 & $3.82(3)$ & $3.58(3)$ & $10.95(7)$ \\
\hline 31.1 & $3.86(3)$ & $3.51(2)$ & $10.97(3)$ \\
\hline 32.3 & $3.87(3)$ & $3.48(2)$ & $10.89(3)$ \\
\hline 34.7 & $3.91(9)$ & $3.48(5)$ & $10.87(7)$ \\
\hline 37.0 & $3.82(4)$ & $3.50(2)$ & $10.85(2)$ \\
\hline 40.0 & $3.81(4)$ & $3.49(2)$ & $10.82(3)$ \\
\hline 41.6 & $3.79(4)$ & $3.49(3)$ & $10.79(3)$ \\
\hline 42.4 & $3.79(4)$ & $3.47(2)$ & $10.75(2)$ \\
\hline 50.8 & $3.59(15)$ & $3.46(15)$ & $10.9(3)$ \\
\hline
\end{tabular}

${ }^{\text {a }}$ Determined by precision diffractometry.
TABLE IV. Lattice constant $a_{0}$ of cubic UP at ambient pressure and room temperature.

\begin{tabular}{llcl}
\hline \hline$a_{0}(\AA)$ & Compos. & Ref. & \multicolumn{1}{c}{ Remarks } \\
\hline 5.5844 & & this work & \\
5.584 & UP $_{0.98}$ & 14 & \\
5.586 & UP $_{0.99}$ & 14 & \\
5.589 & UP $_{1.00}$ & 14 & \\
$5.5888(1)$ & UP & 15 & \\
$5.5888(2)$ & UP & 16 & $\begin{array}{l}\text { Prep. by diffusion } \\
\text { process }\end{array}$ \\
$5.5882(2)$ & UP & 16 & $\begin{array}{l}\text { Prep. by exothermal } \\
\text { process } \\
5.46\end{array}$ \\
\hline \hline
\end{tabular}

Figure 3 shows the relative volume of UP as a function of pressure. The curve for the cubic phase has been calculated from the equation of state. It is seen that the volume versus pressure is described by a continuous curve in the whole pressure range. There are no volume discontinuities at the phase transitions.

\section{B. Influence of the electronic structure}

The ordering temperatures and magnetic moments of metallic compounds are sensitive to volume in a manner summarized in the Hill plots. ${ }^{19}$ For uranium compounds there is a transition region for a U-U distance of about $3.4 \AA$, below which one expects nonmagnetic behavior and delocalization of the $5 f$ electrons.

The U-U separation in cubic UP is $3.95 \AA$ at ambient pressure and $3.85 \AA$ when the transition to rhombohedral UP II occurs at $10 \mathrm{GPa}$. The U-U distance has decreased to $3.76 \AA$ at $28 \mathrm{GPa}$ where the transition to orthorhombic UP III takes place. At the maximum pressure, 51 $\mathrm{GPa}$, in the present work the U-U separation is 3.46-3.59 $\AA$ measured in the (001) plane of the orthorhombic unit cell. Thus the U-U distance is above the

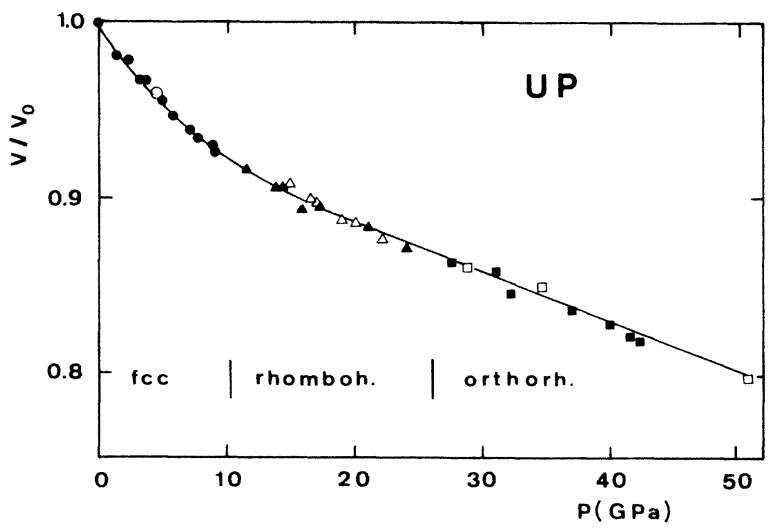

FIG. 3. Relative volume of UP as a function of pressure. The curve for the fcc phase $(<10 \mathrm{GPa})$ has been calculated from the equation of state. The symbol are the same as in Fig. 2. 
TABLE V. The bulk modulus $B_{0}$ of cubic UP and its pressure derivative $B_{0}^{\prime}$ at ambient pressure. The uncertainties, given in parentheses, are the standard deviations of the least-squares fit to the equation state.

\begin{tabular}{rccc}
\hline \hline$B_{0}(\mathrm{GPa})$ & $B_{0}^{\prime}$ & Ref. & $\begin{array}{c}\text { Pressure } \\
\text { region }\end{array}$ \\
\hline $102(4)$ & $4.0(8)$ & this work & $p \leq 10 \mathrm{GPa}$ \\
$250(10)$ & $<0$ & 1 & $p \leq 18 \mathrm{GPa}$ \\
$102(3)$ & $4.7(5)$ & 2 & $p \leq 25 \mathrm{GPa}$ \\
107 & & 17 & Theor. value \\
\hline
\end{tabular}

critical value $3.4 \AA$ in the whole pressure range investigated.

In comparison, $\mathrm{UC}$ and $\mathrm{UN}$ have the smallest anions and therefore the smallest U-U separation of the uranium rocksalt-structure compounds. High-pressure studies have shown that UC (Ref. 20) and UN (Ref. 4) transform to distorted fcc structures when the U-U distance is 3.34-3.38 $\AA$, i.e., just below the critical value in the Hill plot. The transitions are of first order with a volume collapse of $3-5 \%$. The corresponding thorium compounds, ThC (Ref. 21) and ThN (Ref. 22), have no phase transformations and remain cubic in the same pressure range. UAs, USe, USb, and UTe have larger anions and correspondingly larger U-U separation. These compounds are all found to transform to the CsCl-type structure $(B 2)$ at high pressure with a volume collapse of about $10 \% .^{23}$ The same transformations are found for the corresponding thorium compounds as well as ThP. ${ }^{23}$

From high-pressure studies on the rare-earth metals it is now understood that the $s$ and $d$ electrons are responsible for high-symmetry structures (hcp, Sm type, dhcp, and $\mathrm{fcc}$ ), whereas the $f$ electrons tend to favor distorted structures as seen in the case of cerium (e.g., Ref. 24).

A similar correlation between crystal structure and electronic structure has been found for the actinide met- als. Thorium has the high-symmetric fcc structure in accordance with the $5 f$ band of thorium being essentially unoccupied. Uranium, on the other hand, has about three $5 f$ electrons which are nonlocalized (itinerant) and contribute to the metallic bonding. Consequently, uranium has a low-symmetric structure (orthorhombic $\alpha$-U).

Proceeding along the actinide series in the Periodic Table it is not until americium that one again finds highsymmetric structures similar to those of the rare earths. In the heavy actinides the $5 f$ electrons are localized and do not participate in the metallic bonding.

From the evidence above we conclude that the transformation to the CsCl-type structure observed for the large anion uranium and thorium compounds is determined by the $7 s$ and $6 d$ electrons of the actinide, possibly together with the electrons of the ligand. The transformation of the small-anion uranium compounds is determined by the influence of the $5 f$ electrons. The structural stability of the small-anion thorium compounds is explained by their low $5 f$ occupation number.

The phase transformations of UP reported in the present work do not fall into one or the other of the categories discussed above. The distorted structures of UP II and UP III indicate an influence of the $f$ electrons. However, the U-U separation is above the critical value of the Hill plot, indicating that the $s$ and $d$ electrons also have some influence on the transitions. Absorption-edge measurements at high pressure would be useful to determine possible valence changes in uranium at the phase transformations of UP.

\section{ACKNOWLEDGMENTS}

We wish to express our thanks to DESY-HASYLAB for the permission to use the synchrotron-radiation facility. Financial support from the Danish Natural Science Research Council is gratefully acknowledged.
'S. N. Vaidya, C. Karunakaran, M. D. Karkhanavala, and R. Krishnan, J. Nucl. Mater. 60, 339 (1976).

${ }^{2}$ J. M. Leger, K. Oki, A. M. Redon, I. Vedel, J. Rossat-Mignod, and O. Vogt, Phys. Rev. B 33, 7205 (1986); I. Vedel, K. Oki, A. M. Redon, R. M. Leger, J. Rossat-Mignod, and O. Vogt, J. Less-Common Met. 121, 157 (1986); I. Vedel, A. M. Redon, and J. M. Leger, Physica B + C 144B, 61 (1986).

${ }^{3}$ J. Staun Olsen, S. Steenstrup, L. Gerward, U. Benedict, J. C. Spirlet, and G. D. Andreetti, J. Less-Common Met. 98, 291 (1984), and unpublished.

${ }^{4}$ J. Staun Olsen, L. Gerward, and U. Benedict, J. Appl. Crystallogr. 18, 37 (1985).

${ }^{5} \mathrm{~K}$. Mattenberger, L. Scherrer, and O. Vogt, J. Cryst. Growth 67, 467 (1984).

${ }^{6}$ B. Okai, O. Shimomura, and I. Fujishiro, Physica B + C 139\&140B, 799 (1986).

${ }^{7}$ R. Le Sar, S. A. Ekberg, L. H. Jones, R. L. Mills, L. A. Schwalbe, and D. Schiferl, Solid State Commun. 32, 131 (1979).
${ }^{8}$ H. K. Mao, P. M. Bell, J. Shaner, and D. J. Steinberg, J. Appl. Phys. 49, 3276 (1978).

${ }^{9}$ P. E. Werner, Ark. Kemi 31, 513 (1969).

${ }^{10}$ S. Steenstrup, Aust. J. Phys. 38, 319 (1985).

${ }^{11}$ L. Gerward, J. Staun Olsen, and U. Benedict, Physica B + C 144B, 72 (1986).

${ }^{12}$ S. K. Sikka and V. Vijayakumar, Physica B + C 144B, 23 (1986).

${ }^{13}$ See, for example, C. S. Menoni and I. L. Spain, in High Pressure Measurement Techniques, edited by G. N. Peggs (Applied Science, London, 1983), p. 133.

${ }^{14}$ Y. Baskin, J. Am. Ceram. Soc. 49, 541 (1966).

${ }^{15}$ O. L. Kruger and J. B. Moser, J. Phys. Chem. Solids 28, 2321 (1967).

16J. L. Driscoll and P. E. Evans, J. Nucl. Mater. 28, 311 (1968).

${ }^{17}$ M. S. S. Brooks, J. Phys. F 14, 653 (1984).

${ }^{18}$ A. K. Singh and C. Balasingh, J. Appl. Phys. 48, 5338 (1977).

${ }^{19}$ H. H. Hill, Nucl. Metall. 17, 2 (1971).

${ }^{20}$ J. Staun Olsen, L. Gerward, U. Benedict, J.-P. Itié, and K. 
Richter, J. Less-Common Met. 121, 445 (1986).

${ }^{21}$ L. Gerward, J. Staun Olsen, U. Benedict, J.-P. Itié, and J. C. Spirlet, J. Appl. Crystallogr. 19, 308 (1986).

${ }^{22}$ L. Gerward, J. Staun Olsen, U. Benedict, J.-P. Itié, and J. C.
Spirlet, J. Appl. Crystallogr. 18, 339 (1985).

${ }^{23}$ J. Staun Olsen, L. Gerward, S. Dabos, U. Benedict, O. Vogt, and J. C. Spirlet (unpublished).

${ }^{24}$ B. Johansson, J. Magn. Magn. Mater. 47\&48, 231 (1985). 